\title{
Iberian and Macaronesian Vegetation Information System (SIVIM)
}

\author{
Xavier Font Castell, Idoia Biurrun, Federico Fernández-Gonzalez \& Carmen Lence
}

\begin{abstract}
SIVIM (Sistema de Información de la Vegetación Ibérica y Macaronésica; GIVD ID EU-00-004) is an information system designed for capturing, hosting, editing, analysing and exporting georeferenced plot data of Iberian and Macaronesian vegetation. It currently hosts 125,000 relevés, mainly from the northern half of the Iberian Peninsula and the Balearic Islands, and will grow to 150,000 relevés in the near future. SIVIM has been conceived to offer direct and free on-line access to relevés, tables, as well as to floristic, syntaxonomical and bibliographical records. The system also offers on-line software for edition and analysis of vegetation data (http://www.b-vegana.info).
\end{abstract}

Keywords: biodiversity informatics; floristic databank; relevé; vegetation databank.

Iberian and Macaronesian Vegetation Information System (SIVIM)

Scope: SIVIM is an Iberian and Macaronesian Vegetation Information System that aims to host about 125.000 relevés mainly from the Iberian Peninsula and the Balearic and Canary Islands.

Status: ongoing capture

Period: $1926-2010$

Database manager(s): Xavier Font Castell (xfont@ub.edu)

Owner: [NA]

Web address: http://www.sivim.info

Availability: free online

Online upload: yes

Online search: yes

Database format(s): DB2 9.5 (Express).

Export format(s): XML VegAna

Publication: Font, X.; Rodríguez-Rojo, M.P.; Acedo, C.; Biurrun, I.; Fernández-González, F.; Lence, C. Loidi, j. \& Ninot, J.M. (2010). SIVIM: an on-line database of Iberian and Macaronesian vegetation. Waldökologie, Landschaftsforschung und Naturschutz 8: 15-22.

Plot type(s): normal plots

Non-overlapping plots: 125,000

Plot-size range: $1-400 \mathrm{~m}^{2}$

Total plot observations: 125,000

Estimate of existing plots: 160,000

Completeness: $78 \%$

Countries: AD: 0.1\%; ES: $80.0 \%$; FR: $2.9 \%$; PT: $17.0 \%$

Forest: [NA] - Non-forest: [NA]

Guilds: all vascular plants: 100\%; bryophytes (terricolous or aquatic): 50\%; lichens (terricolous or aquatic): $30 \%$; algae (terricolous or aquatic): $1 \%$ Environmental data: [NA]

Performance measure(s): presence/absence only: $1 \%$

Geographic localisation: point coordinates less precise than GPS, up to $1 \mathrm{~km}: 5 \%$; political units or only on a coarser scale (>10 km): $100 \%$ Sampling periods: $[N A]$

Information as of 2012-07-12; further details and future updates available from http://www.givd.info/ID/EU-00-004

Xavier Font Castell* (xfont@ub.edu)

Department of Plant Biology, University of Barcelona, Avda. Diagonal 645, 08028 Barcelona, SPAIN

Idoia Biurrunidoia.(biurrun@ehu.es)

Department of Plant Biology and Ecology; University of Basque Country - UPV/EHU, 48080 Bilbao, SPAIN

Federico Fernández-González (Federico.Fdez@uclm.es)

Institute of Environmental Sciences; University of Castilla-La Mancha; 45071 Toledo, SPAIN

Carmen Lence (carmenlence@unileon.es)

Department of Environmental Management and Biodiversity; University of León; 24072 León, SPAIN

*Corresponding author 